\title{
VIABILIDADE DE COMPÓSITOS POLIMÉRICOS DE POLIPROPILENO REFORÇADOS COM FIBRA DE BAMBU
}

\author{
VIABILITY OF POLYPROPYLENE POLYMER COMPOUNDS REINFORCED WITH \\ BAMBOO FIBER \&
}

\author{
VIABILIDAD DE LOS COMPUESTOS POLÍMEROS DE POLIPROPILENO \\ REFORZADOS CON FIBRA DE BAMBÚ \&
}

Recebido em: 01/11/2021 - Aprovado em: 19/11/2021 - Publicado em: 29/11/2021

doi) http://dx.doi.org/10.18011/bioeng2021v15n4p511-522

Diogo Antonio Correa Gomes' (diogo.gomes2@estudante.ufla.br)

Eduardo Hélio de Novais Miranda' (eduardohelio013@gmail.com.br)

Ana Carolina Corrêa Furtini' ${ }^{1}$ (carol.furtini@gmail.com)

Carolina Aparecida dos Santos ${ }^{1}$ (carolinaapnep@gmail.com)

Mariane Duarte Resende ${ }^{1}$ (marianeduarte-@hotmail.com)

Denisse Concepción Vega Villarruel ${ }^{1}$ (denisse.villarruel@estudante.ufla.br)

José Benedito Guimarães Júnior ${ }^{1}$ (jose.guimaraes@ufla.br)

1 Universidade Federal Lavras. Lavras, Minas Gerais, Brasil

\section{RESUMO}

O objetivo desta pesquisa foi a avaliação das propriedades mecânicas de compósitos poliméricos de matriz de polipropileno com adição de fibras de bambu (Dendrocalamus strictus). Para tanto, o bambu foi processado em um moinho de martelos. As partículas resultantes foram selecionadas granulometricamente, as quais foram caracterizados através dos ensaios de umidade básica, densidade aparente e total de extrativos, lignina e cinzas e secas até a umidade de $3 \%$. Já o polipropileno foi adquirido no comércio local. Quatro corpos de prova de cada tratamento $(80 \%$ de polipropileno e $20 \%$ de fibras de bambu; $100 \%$ de polipropileno) foram então moldados a partir destas duas matériasprimas em uma caixa formadora, conformados à quente e submetidos aos ensaios de flexão estática, a fim de obtenção do módulo de elasticidade (MOE) e módulo de ruptura (MOR). A influência da adição de fibras de bambu em matrizes de polipropileno foi verificada através de uma análise estatística de análise de variância (ANAVA), a $5 \%$ de significância. Os resultados demonstraram que as fibras de bambu apresentaram altos valores de lignina, holocelulose, densidade e extrativos, concomitante à valores de cinzas e umidade compatíveis a outras pesquisas. Além disso, as propriedades dos compósitos contendo adição deste material natural não apresentaram valores estatisticamente diferentes em relação às amostras referência. Os valores de MOE e MOR absolutos, entretanto, sofreram tendências respectivas de aumento e redução com a incorporação de fibra de bambu. Não foi possível comprovar, portanto, a viabilidade deste nível de adição de fibras de bambu em compósitos de matriz de polipropileno e recomenda-se, em trabalhos futuros, a realização de pré-tratamentos nas fibras e/ou adição de diferentes níveis destes materiais lignocelulósicos no compósito.

Palavras-chave: Biocompósitos, Fibras vegetais; Sustentabilidade. 


\section{INTRODUÇÃO}

A utilização de reforços renováveis em compósitos apresenta inúmeros desafios devido às características desses materiais, como sua alta higroscopicidade, que podem apresentar tendência à absorção de água, sua fragilidade á ataques de agentes xilófagos, e suas características químicas que podem apresentar diferentes proporções de celulose, hemicelulose e lignina e podem limitar suas aplicações.

Apesar destes desafios, as fibras vegetais têm atraído à atenção de cientistas e pesquisadores de materiais para a produção de compósitos poliméricos devido a sua abundância, baixo custo, baixa densidade, facilidade de obtenção e manuseio, baixa abrasão de equipamentos e moldes, biodegradabilidade e renovabilidade. Neste sentido, tais elementos vêm se apresentando como boa alternativa à substituição às fibras sintéticas, tais como, fibras de vidro, de carbono e de aramida, que causam efeitos indesejados no meio ambiente. (VENKATARAJAN et al., 2021).

Uma das fibras utilizadas para este propósito são as fibras de bambu. Isso ocorre, pois, o bambu é um material natural, ecológico, de alta resistência e tenacidade. É amplamente utilizado na indústria moveleira, têxtil e de construção devido ao seu ciclo de crescimento curto (WU et al., 2021). As fibras formadas por esta planta concedem ainda uma boa resistência aos materiais que as contém, além de maior rigidez e força, principalmente, quando comparadas às fibras de madeiras folhosas e coníferas (GUIMARÃES JÚNIOR et al.,2010).

O bambu, diante destas vantagens, vem sendo utilizado como matéria-prima na geração de vários tipos de materiais compósitos, tais como: madeira laminada de bambu, OSB (Oriented Strand Board), compósitos poliméricos, dentre outros. (WU et al., 2021; KUMARI et al., 2020). Estudos dos impactos da adição de fibras de bambu, entretanto, em matrizes poliméricas são ainda insipientes e, por isso, novas pesquisas são necessárias a fim de estudos mais abrangentes destes impactos e, frente a resultados positivos, contribuir para a popularização deste produto que ainda sofre paradigmas sociais quanto ao uso em larga escala.

Face a todas essas questões expostas anteriormente, o objetivo deste projeto de pesquisa é a avaliação da adição fibra vegetal de bambu (Dendrocalamus strictus) como elemento de reforço em matrizes poliméricas de polipropileno. 
O bambu utilizado na pesquisa foi da espécie Dendrocalamus strictus. Esse material foi obtido a partir de plantio experimental no campus da Universidade Federal de Lavras (UFLA). Já o polipropileno utilizado foi adquirido no comércio de Lavras, Minas Gerais.

Para a preparação das partículas de bambu, inicialmente seus colmos foram cortados em ripas, transformados em cavacos e processados em um moinho de martelos de alta rotação Lucato (Limeira, São Paulo, Brasil). Obteve-se assim, por redução, partículas, que foram selecionadas para os próximos procedimentos, com o uso de um peneirador vibratório A Bronzinox (São Paulo, Brasil), como as retidas na peneira de 100 mesh.

A análise deste material lignocelulósico resultante foi determinada através dos seguintes ensaios: umidade básica, densidade aparente - NBR 14810-2 (ABNT, 2018), total extrativos - NBR 14853 (ABNT, 2010), lignina insolúvel em ácido - NBR 7989 (ABNT, 2010) e teor de cinzas - NBR 13999 (ABNT, 2017). O teor de holocelulose também pode ser calculado através da diferença percentual dos componentes anteriores.

Antes da produção dos painéis, as partículas foram secas até a umidade $3 \% \mathrm{em}$ uma estufa Marconi MA035 (Piracicaba, São Paulo, Brasil), por um período de 24 horas a temperatura $103 \pm 2{ }^{\circ} \mathrm{C}$ (NELSON et al., 2013).

Para a confecção dos corpos de prova, utilizou-se caixa formadora de $20 \times 12 \times 2 \mathrm{~cm}$ (comprimento, largura, espessura). Inicialmente, apenas o polipropileno foi adicionado na extrusora para fins de limpeza do aparelho. Em seguida, iniciou-se o processo de moldagem de quatro corpos de prova para cada tratamento ( $80 \%$ de polipropileno e $20 \%$ de fibras de bambu; $100 \%$ de polipropileno).

Para a produção dos moldes foi utilizado uma extrusora dupla rosca NZPhilpolymer SJSL-20 (São Roque, São Paulo, Brasil). Tal extrusora é composta por 6 zonas de aquecimento além da saída da matriz. As temperaturas utilizadas em cada zona foram: $120{ }^{\circ} \mathrm{C}$ para a primeira zona, $140 \stackrel{\circ}{\mathrm{C}}$ para a segunda zona, $160{ }^{\circ} \mathrm{C}$ para terceira zona, $170 \stackrel{\circ}{\circ}$ para a quarta zona, $180 \stackrel{\circ}{\circ}$ para a quinta e sexta zona e para na saída da matriz. A extrusão foi feita com uma velocidade de alimentação de 6,1 rpm e velocidade da dupla rosca de $50 \mathrm{rpm}$. Os corpos de prova acondicionados em repouso por 24 horas antes da realização dos próximos procedimentos. 
Para o ensaio de flexão foram então confeccionados corpos de prova utilizando uma prensa e um molde metálico. Em primeiro momento as formas foram preenchidas a fim de redução dos espaços vazios. Em seguida, realizou-se o processo de pré-prensagem em uma prensa hidráulica Marconi MA 098 (Piracicaba, São Paulo, Brasil), que consistiu, basicamente, em três etapas:

- $\quad$ Prensagem por 5 minutos a $150^{\circ} \mathrm{C}$ sem nenhuma pressão;

- $\quad$ Prensagem durante 5 segundos com pressão de $3 \mathrm{MPa}$;

- $\quad$ Prensagem por 5 minutos a $150^{\circ} \mathrm{C}$ sem nenhuma pressão.

Posteriormente, os compósitos foram prensados por um tempo de 20 segundos, sob pressão de $12 \mathrm{MPa}$ a uma temperatura de $150^{\circ} \mathrm{C}$ e, em seguida, armazenados em uma sala climatizada a temperatura de $24^{\circ} \mathrm{C}$ até a realização do ensaio mecânico.

As amostras foram então ensaiadas à flexão estática de três pontos em uma máquina de ensaios universal localizada na UEPAM (Unidade Experimental em Painéis de Madeira) - UFLA. A partir das curvas obtidas, tornou-se possível o cálculo do módulo de elasticidade (MOE) e módulo de ruptura (MOR) (ASTM D-790-15).

A influência da adição de fibras de bambu nas propriedades mecânicas de compósitos de matriz de polipropileno foi verificada através de uma análise estatística de análise de variância (ANAVA), a 5 \% de significância. O programa computacional utilizado para este fim foi Sisvar (Departamento de Ciências Exatas, Lavras, Minas Gerais).

\section{RESULTADOS E DISCUSSÃO}

A Tabela 1 apresenta os resultados observados para a caracterização das fibras de bambu.

Tabela 1 - Caracterização física e química das fibras de bambu.

\begin{tabular}{cc}
\hline Constituintes químicos & Valores \\
\hline Umidade básica (\%) & 8,20 \\
Densidade aparente $\left(\mathrm{g} / \mathrm{cm}^{3}\right)$ & 0,16 \\
Extrativos (\%) & 23,25 \\
Lignina (\%) & 18,01 \\
Cinzas (\%) & 0,22 \\
Holocelulose (\%) & 28,52 \\
\hline
\end{tabular}

Fonte: Autores, 2021. 
A caracterização química do material foi compatível com os valores difundidos na literatura. Já na avaliação da densidade aparente, valor obtido por Kumari et al. (2020) foi de $0,075 \mathrm{~g} / \mathrm{cm}^{3}$, inferior ao presente estudo.

O teor de umidade encontrado na fibra do bambu foi de 8,20\%. De acordo com Silva et al. (2006), a umidade encontrada nos resíduos de soja foi de 8,40 \%. Nesses dois casos, os valores de umidade da fibra foram próximos e altos. Esse grande teor de umidade pode provocar uma estrutura de microfraturas na superfície de compósitos poliméricos a serem formados.

Os valores encontrados para lignina e holocelulose foram, por outro lado, superiores ao encontrado por Sanchez-Echeverri et al. (2021) que obtiveram 9,70 \% para a lignina e $13,10 \%$ para holocelulose. Essa diferença nesses valores se deu em decorrência da falta de pré-tratamentos na fibra que removem, aproximadamente, 37,00 \% da lignina e 34,00 \% de holocelulose.

Para os valores de extrativos totais, Martins et al. (2018) encontraram um teor superior $(26,72 \%)$ para o resíduo de soja. Nas fibras de bambu foi encontrado um valor de 23,25 \%, valor absolutamente inferior, mas também alto. Segundo Shebani et al. (2018), um teor baixo de extrativos pode ser positivo para a adição de materiais lignocelulósicos com compósitos poliméricos, fato não observado nesta presente pesquisa.

Os teores de cinza encontrados por Brito (1979) nos resíduos de soja foram altos. De acordo com o autor, isto é um fator prejudicial, em alguns casos, até limitante ao uso de materiais lignocelulósicos em compósitos de alto rendimento. Os valores encontrados por Silva et al. (2006), porém, para esta propriedade foi de 8,90\%. O bambu, em comparação a estes estudos, apresentou um baixo teor de cinzas $(0,22 \%)$, e isto pode significar um fator positivo para sua aplicação tecnológica.

Depois de caracterizadas "in natura", as fibras foram avaliadas como material de reforço em matrizes de polipropileno. A elevada dispersão das fibras foi identificada como um fator importante que influencia as propriedades dos compostos resultantes. Durante a moldagem, a distribuição das fibras não foi uniforme; no entanto, a dispersão uniforme é um desafio para fibras longas devido à sua maior tendência para aglomerar (HAO et al., 2021).

Uma boa distribuição das fibras no compósito tem grande influência nos valores de módulo de elasticidade, para cada tratamento, que estão apresentados na Figura 1. 
Figura 1 - Dados de módulo de elasticidade (MOE) obtidos.

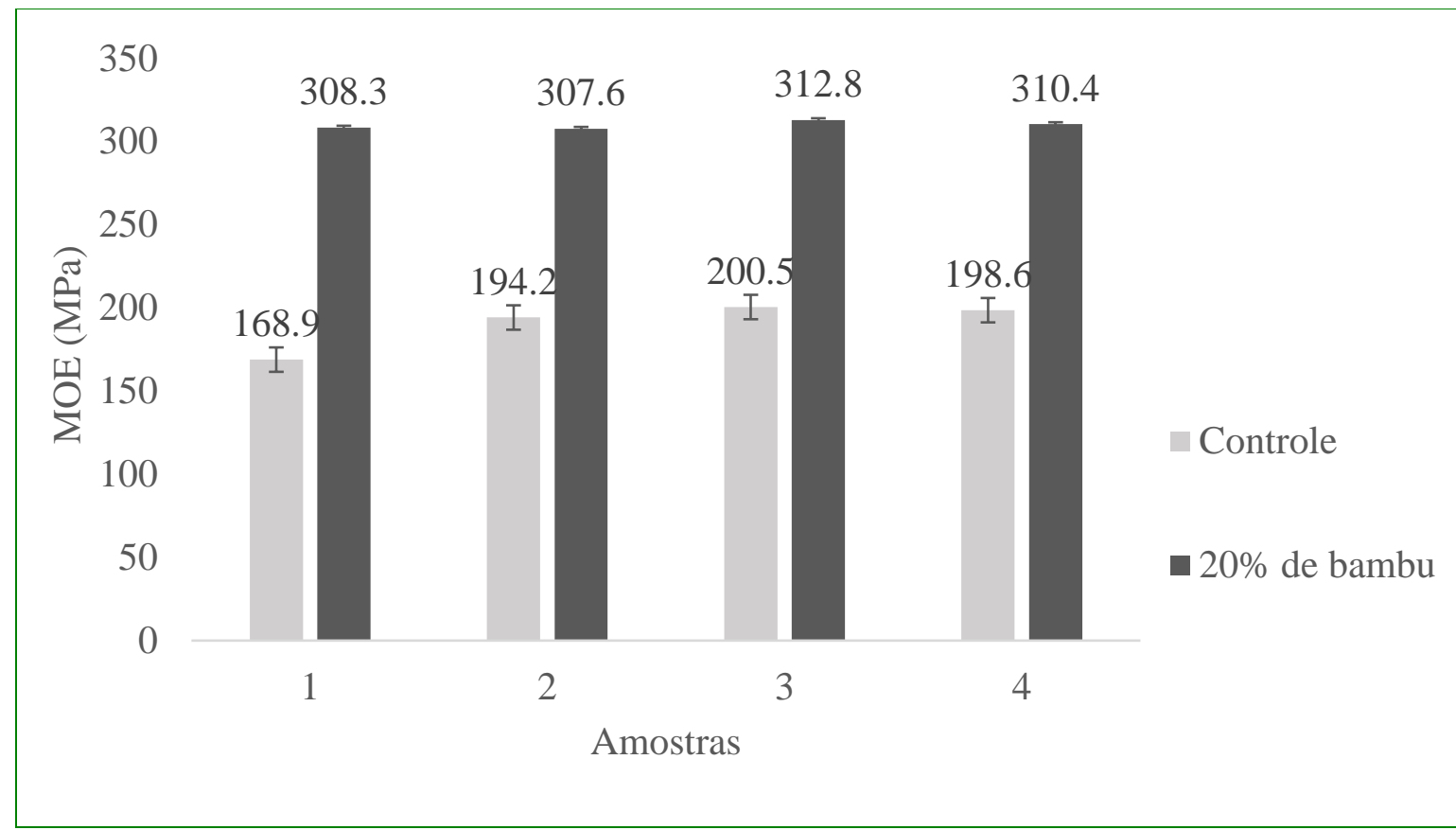

Fonte: Autores, 2021.

A partir do teste ANAVA, com nível de significância de $5 \%$, observou-se, todavia, que não houve diferença estatística entre os dados de MOE obtidos pelos dois tratamentos. Os valores de MOE obtidos dos compósitos com $20 \%$ de material lignocelulósico apresentaram inclusive, em todos os ensaios, valores absolutos superiores em comparação às amostras de referência, o que pode estar relacionado a grande presença de lignina nestes elementos naturais.

$\mathrm{Na}$ pesquisa de Targa, 2011, os valores de MOE obtidos foram superiores ao encontrados neste trabalho, o que pode ser explicado pelos diferentes níveis de adição de fibras de bambu nos produtos de polipropileno.

Os valores do módulo de ruptura obtidos, porém, estão apresentados na Figura 2. 
Figura 2 - Valores do módulo de ruptura (MOR) obtidos.

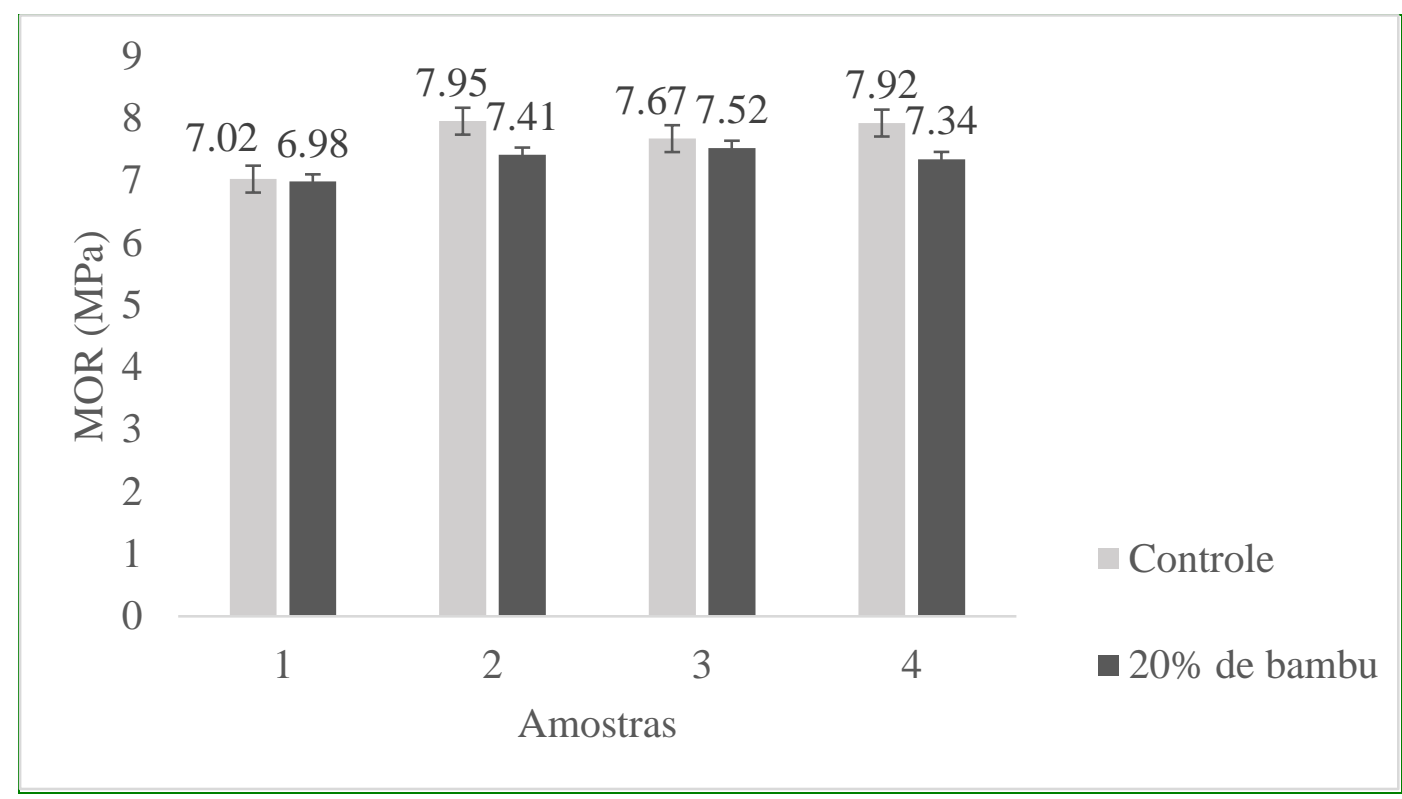

Fonte: Autores, 2021.

Discute-se que também não houve diferença significativa entre os tratamentos para a propriedade de MOR, segundo o teste ANAVA, com nível de significância de $5 \%$. Os valores de MOR absolutos, entretanto, sofreram leves reduções com a incorporação de fibra de bambu. Esse comportamento pode ser explicado devido à possíveis descontinuidades na matriz polimérica produzidas pela presença de fibras, impedindo a distribuição e a transferência da carga aplicada, e assim, diminuindo a resistência dos compósitos (KUMARI et al., 2020).

\section{CONCLUSÃO}

O objetivo desta pesquisa foi a avaliação da adição fibra vegetal de bambu (Dendrocalamus strictus) como elemento de reforço em matrizes poliméricas de polipropileno.

A partir dos resultados encontrados, as fibras de bambu apresentaram altos valores de lignina, holocelulose, densidade e extrativos, além de valores de cinzas e umidade compatíveis à outras literaturas.

As propriedades mecânicas das amostras de polipropileno com fibras de bambu, entretanto, não apresentaram valores estatisticamente diferentes entre os tratamentos 
realizados. Os valores de MOE e MOR absolutos, entretanto, sofreram tendências respectivas de aumento e redução com a incorporação de fibra de bambu.

Portanto, apesar de apresentar alguns pontos positivos, não foi possível verificar a viabilidade da adição de fibras de bambu em matrizes de polipropileno e novos estudos são recomendados para uma melhor análise destes biocompósitos. Recomenda-se, neste sentido pesquisas utilizando-se, por exemplo, o pré-tratamento de fibras e/ou adição de diferentes níveis destes materiais lignocelulósicos no compósito a fim de confecção de produtos que atendam as normativas e popularizem o uso deste material sustentável.

\section{REFERÊNCIAS}

ASSOCIAÇÃO BRASILEIRA DE NORMAS TÉCNICAS. ABNT NBR 7989. Pasta celulósica e madeira - Determinação de lignina insolúvel em ácido. Rio de Janeiro, 2010.

ASSOCIAÇÃO BRASILEIRA DE NORMAS TÉCNICAS. ABNT NBR 11941. Madeira Determinação da densidade básica. Rio de Janeiro, 2003.

ASSOCIAÇÃO BRASILEIRA DE NORMAS TÉCNICAS. ABNT NBR 13999. Papel, cartão, pastas celulósicas e madeira - Determinação do resíduo (cinza) após a incineração a $525^{\circ} \mathrm{C}$. Rio de Janeiro, 2017.

ASSOCIAÇÃO BRASILEIRA DE NORMAS TÉCNICAS. ABNT NBR 14853. Madeira Determinação do material solúvel em etanol-tolueno e em diclorometano e em acetona. Rio de Janeiro, 2010.

ASTM - AMERICAN SOCIETY FOR TESTING AND MATERIALS. ASTM D790. Standard Test Methods for Flexural Properties of Unreinforced and Reinforced Plastics and Electrical Insulating, West Conshohocken, 2017.

BARRICHELO, L., BRITO, J.O., "Usos diretos e propriedades da madeira para geração de energia", Silvicultura, v. 2, n. 12, pp. 26-28, 1979.

DUNNE R.; DESAI D.; SADIKU R.; JAYARAMUDU, J. A review of natural fibres, their sustainability and automotive applications. Journal of Reinforced. Plastics and Composites, v. 35, n.13, p. 1041-1050, 2016.

FEBRIANTO, F.; JANG.; J.H.; LEE, S. H.; SANTOSA, I. A.; HIDAYAT, W.; KWON, J.H.; KIM, N.H. Effect of Bamboo Species and Resin Content on Properties of Oriented Strand Board Prepared from Steam-treated Bamboo Strands. Bioresources, v. 10, n. 2, p. 2642 - 2655, 2015.

GUIMARÃES JÚNIOR, M; NOVACK, K. M; BOTARO, V. R. Caracterização anatômica da fibra de bambu (Bambusa vulgaris) visando sua utilização em compósitos poliméricos.

Revista Iberoamericana de polímeros, v. 11, n. 7, p. 442-456, 2010. 
HAO, J.; YI, Xin; ZONG, G.; SONG, Y.; WANG, W.; CHENG, H.; WANG, G. Fabrication of long bamboo fiber-reinforced thermoplastic composite by extrusion and improvement of its properties. Industrial Crops and Products, v. 173, p. 114120, 2021.

KHALIL, H. P. S. A.; BHAT, I. U. H.; JAWAID, M.; ZAIDON, A.; HERMAWAN, D.; HADI, Y. S. Bamboo fibre reinforced biocomposites: a review. Materials \& Design, v. 42, p. 353368, 2012.

MARTINS, E.H, VILELA, A.P., MENDES, R.F., et al. Soybean waste in particleboard production. Ciência e Agrotecnologia, v. 42, n. 2, p. 186-194, 2018

KUMARI, S.; KUMAR, R.; RAI, B.; SIROHI, S.; KUMAR, G. Study on the modification of polyester resin bamboo fiber-based composite with euphorbia coagulum and their effect on mechanical and thermal properties. Journal of Composite Materials, v. 54, p.24, 2020.

NELSON, P. M; NASCIMENTO, E. M.; NISGOSKI, Silvana; MAGALHÃES, W, L. E.; NETO, C. S; AZEVEDO, E, C. Caracterização física e térmica de compósito de poliuretano derivado de óleo de mamona associado com partículas de bambu. Polímeros: Ciência e Tecnologia, v. 23, n. 2, p. 201-205, 2013.

RAMESH, M. Flax (Linum usitatissimum L.) fibre reinforced polymer composite materials: a review on preparation, properties and prospects. Progress In Materials Science, [S.L.], v. 102, p. 109-166, maio 2019. Elsevier BV. http://dx.doi.org/10.1016/j.pmatsci.2018.12.004.

SANCHEZ-ECHEVERRI, L. A.; GANJIAN, E.; MEDINA-PERILLA, J. A.; QUINTANA, G. C.; SANCHEZ-TORO, J. H.; TYRER, M. Mechanical refining combined with chemical treatment for the processing of Bamboo fibres to produce efficient cement composites. Construction and Building Materials, v. 269, p. 121232, 2021.

SANCHEZ, E. M.S.; CAVANI, C. S.; LEAL, C. V.; SANCHEZ, C. G. Compósito de resina de poliéster insaturado com bagaço de cana-de-açúcar: influência do tratamento das fibras nas propriedades. Polímeros, v. 20, n.3, p. 194-200, 2010.

SCHERER, J F.; BOM, R, P. Determination of shear modulus in bamboo fibers composite in torsion tests, Materials Research Express, v. 6, n. 3, 2018.

SHESHMANI, S., ASHORI, A., FARHANI, F. Effect of extractives on the performance properties of wood flour-polypropylene composites. Journal of Applied Polymer Science 123: 1563-1567, 2012

SILVA, M.S, NAVES, M.M.V., OLIVEIRA, R.B., et al., Composição química e valor protéico do resíduo de soja em relação ao grão de soja. Ciência e Tecnologia de Alimentos,v. 26, n.3, 2006.

SONG, W.; ZHAO, F.; YU, F.; WANG, C.; WEI, W.; ZHANG, S. Interfacial Characterization and Optimal Preparation of Novel Bamboo Plastic Composite Engineering Materials.

Bioresources, v.10, n.3, p. 5049-5070, 2015. 
TARGA, G. N. Compósito reforçado por laminado de bambu com matriz de poliuretano e epóxi: desenvolvimento, produção e caracterização mecânica.

Dissertação (Mestrado em Engenharia Biomateriais) - Universidade Federal de Santa Catarina, Florianópolis, 2011.

XU, Junjie; HAO, Xiaolong; TANG, Wei; ZHOU, Haiyang; CHEN, Lei; GUO, Chuigen; WANG, Qingwen; OU, Rongxian. Mechanical properties, morphology, and creep resistance of ultra-highly filled bamboo fiber/polypropylene composites: effects of filler content and melt flow index of polypropylene. Construction And Building Materials, [S.L.], v. 310, p. 125289, dez. 2021. Elsevier BV.

http://dx.doi.org/10.1016/j.conbuildmat.2021.125289.

WU, Y.; ZHENG, Y.; YANG, F.; YANG, L. Preparation process and characterization of mechanical properties of twisted bamboo spun fiber bundles. Journal of Materials Research and Technology, v. 14, p. 2131-2139, 2021.

WU, J.; YUAN, H.; WANG, W.; WU, Q.; GUAN, X.; LIN, J.; LI, J. Development of laminated bamboo lumber with high bond strength for structural uses by O-2 plasma. Construction and Building Materials, v. 269, n. 1, 2021 


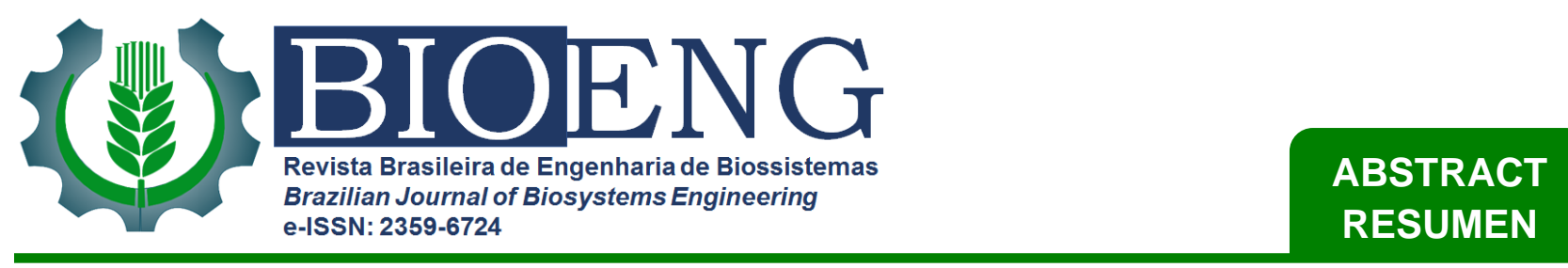

\section{ABSTRACT}

The aim of this research was to evaluate the mechanical properties of polymeric composites made of polypropylene matrix with the addition of bamboo fibers (Dendrocalamus strictus). For this purpose, the bamboo was processed in a hammer mill. The resulting particles were selected by its granulometry, characterized through basic moisture test, bulk density and total of extractives, lignin and ash, and dried to a moisture content of $3 \%$. While, polypropylene was purchased from local businesses. Four specimens from each treatment (80\% polypropylene and $20 \%$ bamboo fibers; $100 \%$ polypropylene) were molded from these two raw materials were shaped in a forming hot box, and subjected to static bending tests, in order to obtain the modulus of elasticity (MOE) and modulus of rupture (MOR). The influence of the addition of bamboo fibers in polypropylene matrices was verified through a statistical analysis of variance (ANOVA) at $5 \%$ significance. The results showed that bamboo fibers presented high values of lignin, holocellulose, density and extractives, concomitant to ash and moisture values compatible with other researches. Furthermore, the properties of composites containing the addition of this natural material did not show statistically different values in relation to the reference samples. The absolute values of MOE and MOR, experienced increasing and decreasing trends with the incorporation of bamboo fiber respectively. Therefore, it has not been possible to demonstrate the viability of this level of bamboo fibers addition in polypropylene matrix composites and it is recommended, in future works, to carry out pretreatments on the fibers and/or addition of different levels of these lignocellulosic materials in the composite.

Keywords: Biocomposites, Vegetable Fibers, Sustainability.

\section{RESUMEN}

El objetivo de esta investigación fue evaluar las propiedades mecánicas de compuestos poliméricos obtenidos a partir de una matriz de polipropileno con la adición de fibras de bambú (Dendrocalamus estricus). Para ello, el bambú fue procesado en un molino de martillos. Las partículas resultantes fueron seleccionadas granulométricamente, caracterizadas mediante pruebas de humedad básica, densidad aparente y total de extractivos, lignina y cenizas, y secadas hasta un contenido de humedad del $3 \%$. Mientras que, el polipropileno, fue adquirido de empresas locales. A continuación, se moldearon cuatro muestras de cada tratamiento ( $80 \%$ polipropileno y $20 \%$ fibras de bambú; 100 $\%$ polipropileno) a partir de estas se moldearon dos materias primas en una caja formadora en caliente y sometidas a pruebas de flexión estática, con el fin de obtener el módulo de elasticidad (MOE) y módulo de ruptura (MOR). La influencia de la adición de fibras de bambú en matrices de polipropileno se verificó mediante un análisis estadístico de varianza (ANOVA) al $5 \%$ de significancia. Los resultados mostraron que las fibras de bambú presentan altos valores de lignina, holocelulosa, densidad y extractivos, concomitantes a valores de ceniza y humedad compatibles con otras investigaciones. Además, las propiedades de los composites que contienen la adición de este material natural no mostraron valores estadísticamente diferentes en relación a las muestras de referencia. Sin embargo, los valores absolutos de MOE y MOR, experimentaron tendencias de aumento y disminución con la incorporación de fibra de bambú, respectivamente. Por tanto, no se ha podido demostrar la viabilidad de este nivel de adición de fibras de bambú en composites de matriz de polipropileno y se recomienda, en trabajos futuros, realizar pretratamientos sobre las fibras y / o adición de diferentes niveles de estos materiales lignocelulósicos en el compuesto.

Palabras clave: Biocomposites, Fibras vegetales; Sustentabilidad. 


\section{LICENÇA DE USO}

Este é um artigo publicado em acesso aberto (Open Access) sob a licença Creative Commons Atribuição 4.0 Internacional (CC BY 4.0), que permite uso, distribuição e reprodução em qualquer meio, desde que o trabalho original seja corretamente citado. Mais informações em: http://creativecommons.org/licenses/by/4.0

\section{CONFLITO DE INTERESSES}

Os autores declaram que não há conflito de interesses neste trabalho.

\section{FINANCIAMENTO}

O presente trabalho não contou com apoio financeiro.

\section{COMO REFERENCIAR}

GOMES, Diogo Antonio Correa et al. Viabilidade de compósitos poliméricos de polipropileno reforçados com fibra de bambu. Revista Brasileira de Engenharia de Biossistemas (Tupã), v. 15, n. 4, p. 511-522, 2021. DOI:

http://dx.doi.org/10.18011/bioeng2021v15n4p511-522.

\section{RESPONSABILIBADE EDITORIAL}

Prof. Dr. Fernando Ferrari Putti ${ }^{1}$, Prof. Dr. Paulo Sérgio Barbosa dos Santos ${ }^{1}$, Prof. Dr. Eduardo Festozo Vicente ${ }^{1}$ e Prof. Dr. Diogo de Lucca Sartori ${ }^{1}$

1 Universidade Estadual Paulista "Júlio de Mesquita Filho", FCE - Faculdade de Ciências e Engenharia, Tupã, SP, Brasil. 